\title{
Condiciones Extremas de Operación en Unidades de Desintegración Catalítica y Multiplicidad de Estados Estacionarios
}

\author{
José R. Hernández-Barajas (1)* y Richart Vázquez-Román(2)
}

(1) Universidad Juárez Autónoma de Tabasco, División de Ciencias Biológicas, Carr. Villahermosa-Cárdenas km 0.5, entronque Bosques de Saloya, CP 86039 Villahermosa, Tabasco-México (e-mail: roberto.hernandez@dacbiol.ujat.mx)

(2) Instituto Tecnológico de Celaya, Departamento de Ingeniería Química,

Av. Tecnológico y García Cubas s/n, CP 38010 Celaya, Gto.-México (e-mail: richart@iqcelaya.itc.mx)

* autor a quien debe ser dirigida la correspondencia

\section{Resumen}

Se ha estudiado y analizado las condiciones extremas de operación en unidades de desintegración catalítica y su efecto en la multiplicidad de estados estacionarios. El modelo propuesto está basado en ecuaciones de conservación de materia y energía y ha permitido demostrar que, para ambos modos de combustión, parcial y total, existen entre uno y cinco estados estacionarios. La multiplicidad más común ocurre cuando existen tres estados estacionarios que se presentan a condiciones típicas de operación. La existencia de un solo estado estacionario es producida por una disminución abrupta en la eficiencia de la fluidización y distribución del aire en el regenerador. Se han determinado los dominios de atracción y las trayectorias dinámicas de cada estado estacionario en ese escenario y se sugiere que se debe tomar en consideración este fenómeno en el diseño de una estrategia de control robusto. Finalmente, se muestra que la existencia de cinco estados estacionarios está relacionada con una disminución de la velocidad de oxidación heterogénea del monóxido de carbono.

Palabras clave: desintegración catalítica, multiplicidad, dinámica, simulación, combustión

\section{Severe Operating Conditions in Catalytic Cracking Units and Multi- plicity of Steady-States}

\begin{abstract}
This paper studies an analyzes the effect of severe operating conditions in catalytic cracking units on multiplicity of steady-states. The model proposed here is based on mass and energy conservative equations and has been able to demonstrate the existence of one to five steady states for both partial and complete combustion regimes. The most common multiplicity is the existence of three steady states that occurs at typical operating conditions. The existence of a unique steady state is produced by an abrupt decrease in both fluidization effectiveness and air distribution inside the regenerator. Attraction domains and dynamic trajectories of each steady state have been determined for this scenario and is suggested that this phenomenon must be taken into account in the design of a robust control strategy. Finally, it is shown that the existence of five steady states is related to a decreasing of the heterogeneous oxidation rate of carbon monoxide.
\end{abstract}

Keywords: catalytic cracking, multiplicity, dynamics, simulation, combustion 


\section{INTRODUCCIÓN}

El proceso de desintegración catalítica en lecho fluidizado, FCC por acrónimo en inglés, convierte fracciones pesadas de petróleo en productos de calidad como gasolina de alto octano y olefinas. En la industria se utilizan diferentes tipos de unidades FCC (UFCC). La Fig. 1 muestra el tipo de unidad que se ha estudiado en este trabajo. En el proceso, la reacción se efectúa en el tubo elevador (riser) con tiempo de contacto corto debido a la alta actividad catalítica. Durante la reacción se genera coque que se deposita sobre el catalizador y es posteriormente quemado en el regenerador para restaurar la actividad catalítica.

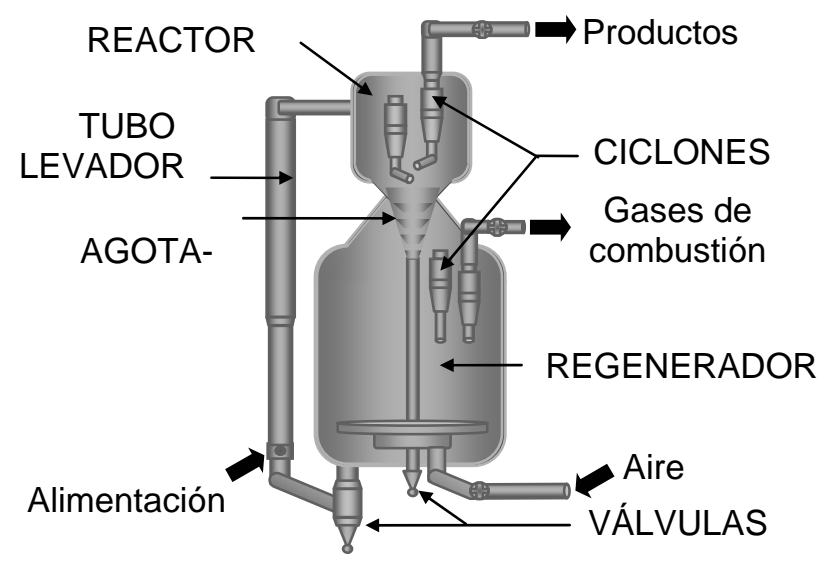

Fig. 1: UFCC típica.

El proceso FCC se considera como el proceso principal de conversión debido a que produce alrededor de la mitad del volumen de gasolinas. Por esta razón, se ha generado un creciente interés en áreas tales como simulación, optimización y control. En particular, la determinación del estado estacionario es relevante porque permite mejorar las estrategias de control. Para una descripción amplia de las estrategias y variables típicas de control, véase Hovd y Skogestad, (1993) y Álvarez-Ramírez et al., (2004). En la literatura pueden encontrarse diversos estudios relacionados a multiplicidad de estados estacionarios en UFCC y cuyos resultados son frecuentemente contradictorios. Iscol (1970) fue el primer autor en considerar la multiplicidad en UFCC. Elnashaie y El-Hennawi (1979) estiman tres estados estacionarios en un intervalo amplio de condiciones de operación en el cual Lee y Kugelman (1973) no pudieron determinar multiplicidad. La existencia de multiplicidad se esclareció con los trabajos de Arandes y de Lasa (1992) y Arbel et al.
(1995). Recientemente, Han y Chung (2001) encontraron dos estados estacionarios estables aunque no reportaron algún estado inestable intermedio.

En este trabajo se utiliza un modelo dinámico basado en ecuaciones de conservación de masa y energía para estudiar el efecto de condiciones de operación extremas en la multiplicidad. La siguiente sección describe el modelo matemático para, posteriormente, presentar la estrategia de solución. Los resultados incluyen el análisis dinámico a lazo abierto para dos casos de estudio estableciéndose las conclusiones en función de los resultados numéricos.

\section{MODELO MATEMÁTICO}

Las etapas principales del proceso involucran la vaporización de la alimentación, la reacción catalítica en el tubo elevador, el agotamiento y la regeneración del catalizador. La etapa de vaporización puede modelarse como un tanque agitado donde no ocurre reacción. La suposición de vaporización instantánea ha sido justificada en diversos estudios, véase $\mathrm{Bu}$ chanan (1994) y Ali y Rohani (1997). Debido a la suposición anterior, un balance de energía global permite conocer la temperatura de mezclado gas-sólido. Por su parte, el tubo elevador puede representarse como un flujo pistón cuya dinámica es mucho más rápida que la del regenerador. Por esta razón resulta apropiado modelar esta sección como un estado pseudo-estacionario. En este estudio se considera que las reacciones catalíticas pueden expresarse a partir de un modelo cinético basado en la técnica del lumping. En principio, las características dinámicas no dependen del modelo cinético seleccionado (Arandes y de Lasa, 1992). Así, en este trabajo se eligió al modelo de 4 grupos (Yen, 1987) por su simplicidad y disponibilidad de información cinética. Las constantes cinéticas del modelo de 4 grupos han sido actualizadas por Han y Chung (2001), ver Tabla 1. Las fracciones masa de cada grupo $y_{i}$, como función de la posición $z$ se expresan por:

$\rho_{g} u_{g} \frac{d y_{i}}{d z}=\frac{\rho_{s}(1-\varepsilon)}{\varepsilon} R_{i} \quad i=1, \ldots, 4$

donde $\rho_{g}, u_{g}$ y $\varepsilon$ representan a la densidad, velocidad y fracción volumétrica de la fase gaseosa, $\rho_{\mathrm{S}}$ es la densidad del catalizador y $R_{i}$ es la velocidad de reacción dada por: 
$R_{i}=\left(\sum_{j=1}^{i-1} k_{j i} y_{j}^{n_{j}}-\left(\sum_{j=i+1}^{4} k_{i j}\right) y_{i}^{n_{i}}\right) \phi$

donde $k_{i j}$ representa a las constantes cinéticas y $n$ es el orden de reacción. La desactivación catalítica $(\phi)$ es una función del coque $(\chi)$ que se forma durante la reacción de acuerdo a un modelo exponencial. Las reacciones catalíticas son de tipo endotérmico por lo que se observa una caída de temperatura con respecto a la posición axial. Por esto, la ecuación de conservación de energía se escribe como:

$C_{P g} u_{g} \beta \frac{d T_{r s}}{d z}=-\sum_{i=1}^{3}\left[y_{i}^{n_{i}} \sum_{j=i+1}^{4} k_{i j}\left(\Delta H_{F}\right)_{j i}\right] \phi$

y $\beta=\frac{\rho_{g}}{\rho_{\mathrm{s}}} \frac{\varepsilon}{(1-\varepsilon)}$

Tabla 1: Factores de frecuencia $(A)$ y energías de activación (E) de las reacciones FCC.

\begin{tabular}{|c|c|c|}
\hline \multicolumn{3}{|c|}{ Desintegración catalítica (Han y Chung, 2001) } \\
\hline Reacción & $A, s^{-1}$ & $\mathrm{E}, \mathrm{kJ} / \mathrm{kgmol}$ \\
\hline Gasóleo a gasolina & 1457.50 & 57359 \\
\hline Gasóleo a gases & 127.59 & 52754 \\
\hline Gasolina a gases & 1.98 & 31820 \\
\hline Gasolina a coque & 256.81 & 65733 \\
\hline \multicolumn{3}{|c|}{ Velocidades de combustión } \\
\hline Expresión y autores & A & $\mathrm{E}, \mathrm{kJ} / \mathrm{kgmol}$ \\
\hline $\begin{array}{l}\text { Coque: } R_{\chi}=k_{\chi} C_{\chi} C_{\mathrm{O}_{2}} \\
\text { Morley y de Lasa, } 1987\end{array}$ & 1.43E8 & 124700 \\
\hline $\begin{array}{l}\mathrm{CO} \text { (homogénea): } \\
\text { Howard et al., } 1973 \\
R_{\mathrm{CO}}=k_{\mathrm{CO}} C_{\mathrm{CO}} C_{\mathrm{O}_{2}}^{0.5} C_{\mathrm{H}_{2} \mathrm{O}}^{0.5}\end{array}$ & 1.30E11 & 124700 \\
\hline $\begin{array}{l}\text { CO (heterogénea): } \\
\text { Schindler y de Lasa, } \\
1984 R_{\mathrm{CO}^{\prime}}=k_{\mathrm{CO}} C_{C O} C_{\mathrm{O}_{2}}^{0.5}\end{array}$ & 248 & 70700 \\
\hline
\end{tabular}

En la actualidad, las reacciones catalíticas se llevan a cabo en el tubo elevador. El reactor es, en realidad, el hogar de los ciclones que separan el catalizador del producto y contiene además la sección de agotamiento donde se desorbe el hidrocarburo atrapado en el catalizador. En el reactor existe una cantidad apreciable de catalizador cuyo tiempo de residencia es de varios minutos. Por esta razón, se ha considerado que esta etapa es relevante en la dinámica del sistema. La acumulación de catalizador $\left(W_{s}\right)$ y productos $\left(W_{g}\right)$ depende de los flujos que entran y salen del reactor:

$$
\begin{aligned}
& \frac{d\left(W_{s}\right)_{r t}}{d t}=\left(F_{s}\right)_{r s}-\left(F_{s}\right)_{r g} \\
& \frac{d\left(W_{g}\right)_{r t}}{d t}=\left(F_{g}\right)_{r s}+\left(F_{v}\right)_{s t}-\left(F_{g}\right)_{m f}
\end{aligned}
$$

donde $t$ es el tiempo, $\left(F_{s}\right)_{r s}$ y $\left(F_{s}\right)_{r g}$ son los flujos de catalizador abandonando el tubo elevador y entrando al regenerador, $\left(F_{g}\right)_{r s}$ y $\left(F_{g}\right)_{m f}$ son los flujos de gas que salen del tubo elevador y entran al fraccionador principal, y $\left(F_{v}\right)_{s t}$ es el flujo de vapor de agotamiento. La temperatura del reactor depende del cambio de temperatura entre el tubo elevador $\left(T_{r s}\right)$ y el reactor $\left(T_{r t}\right)$ y que es causada por la desorción de los productos:

$$
\frac{d T_{r t}}{d t}=\frac{F_{s}\left(T_{r s}-T_{r t}-\Delta T_{r t}\right)}{\left(W_{s}\right)_{r t}}
$$

Por otro lado, es necesario incluir ecuaciones que representen las caídas de presión que ocurren en las distintas válvulas de proceso. Debido a que el reactor opera a presiones bajas y contiene esencialmente hidrocarburos, la presión puede evaluarse a partir de la ley de los gases ideales:

$$
\frac{d P_{r t}}{d t}=P_{r t}\left[\frac{1}{N_{r t}} \frac{d N_{r t}}{d t}+\frac{1}{T_{r t}} \frac{d T_{r t}}{d t}\right]
$$

Los cambios en el número de moles $\left(N_{r t}\right)$ y el volumen del gas $\left(V_{r t}\right)$ son evaluados por:

$$
\begin{aligned}
& \frac{d\left(W_{g}\right)_{r t}}{d t}=M_{g} \frac{d N_{r t}}{d t} \\
& \frac{d V_{r t}}{d t}=\dot{Q}_{r s}-\dot{Q}_{m f}
\end{aligned}
$$

donde $\dot{Q}_{r s}$ y $\dot{Q}_{m f}$ son los flujos volumétricos de gas que salen del tubo elevador y entran al fraccionador. Finalmente, en el reactor realizamos un balance global para el coque. Puesto que el contenido de coque sobre el catalizador no cambia significativamente durante el proceso de agotamiento, entonces los cambios en el contenido de coque dependen únicamente de sus valores a la entrada y salida del reactor:

$\frac{d \chi_{r t}}{d t}=\frac{F_{s}\left(\chi_{r s}-\chi_{r t}\right)}{\left(W_{s}\right)_{r t}}$ 
Por otro lado, el regenerador tiene dos lechos: denso y diluido. En el lecho denso el catalizador se regenera casi completamente y, como en el caso del reactor, en él se encuentra una cantidad significativa de catalizador con elevado tiempo de residencia. Esto implica que el lecho denso afectará a la dinámica del sistema. La acumulación de catalizador y de gases depende del flujo de ambas fases en el lecho:

$$
\begin{aligned}
& \frac{d\left(W_{s}\right)_{r g}}{d t}=\left(F_{s}\right)_{r g}-\left(F_{s}\right)_{r s} \\
& \frac{d\left(W_{g}\right)_{r g}}{d t}=F_{a}-\left(F_{g}\right)_{p l}
\end{aligned}
$$

donde $F_{a}$ y $\left(F_{g}\right)_{p l}$ son los flujos de aire y de gases de combustión. La temperatura del lecho denso es función de la entalpía de combustión del coque y los calores sensibles de las especies interactuando en el regenerador:

$$
\left(W_{s}\right)_{r g} C p_{s} \frac{d T_{d e n}}{d t}=F_{\chi} \Delta H_{\chi}+H_{s}+H_{s c}+H_{\chi}+H_{a}
$$

donde $T_{\text {den }}$ es la temperatura del lecho denso, $F_{\chi}$ y $\Delta H_{\chi}$ son el flujo y entalpía de combustión del coque, $H_{s}$ es el calor sensible del catalizador circulando en el regenerador, $H_{s c}$ el calor sensible del catalizador que se dirige a los ciclones, $H_{\chi}$ el calor sensible del coque en el regenerador, y $H_{a}$ es el cambio entálpico del aire entrante al regenerador. Como el regenerador opera a presiones bajas, los cambios en la presión también se modelan con la ley de gas ideal:

$$
\frac{d P_{r g}}{d t}=P_{r g}\left[\frac{1}{N_{r g}} \frac{d N_{r g}}{d t}+\frac{1}{T_{r g}} \frac{d T_{r g}}{d t}\right]
$$

Los cambios en el número de moles y el volumen del gas son dados por:

$$
\begin{aligned}
& \frac{d\left(W_{g}\right)_{r g}}{d t}=M_{g} \frac{d N_{r g}}{d t} \\
& \frac{d V_{r g}}{d t}=\dot{Q}_{a}-\left(\dot{Q}_{g}\right)_{p l}
\end{aligned}
$$

donde $M_{g}$ es el peso molecular de los gases en el regenerador. Debido a que el coque es quemado en el regenerador, entonces la desaparición del coque por combustión es considerada en el balance de masa:

$$
\frac{d \chi_{r g}}{d t}=\frac{\left(F_{s}\right)_{r t} \chi_{r t}-\left(F_{s}\right)_{r g} \chi_{r g}-F_{\chi}}{\left(W_{s}\right)_{r g}}
$$

El gas de combustión se mueve en flujo pistón ascendiendo entre los espacios del lecho y arrastrando pequeñas cantidades de catalizador hacia el lecho diluido. Las especies que constituyen el gas de combustión se consideran en estado pseudoestacionario ya que su tiempo de residencia es mucho menor que el del catalizador acumulado. La cinética de combustión se rige por las relaciones estequiométricas dadas por Krishna y Parkin (1985). Los balances de masa y energía resultantes han sido presentados en una publicación previa por los autores de este estudio (Hernández et al., 2001). Para completar el modelo, es necesario considerar expresiones para las válvulas. En las UFCC existen dos tipos: válvulas de deslizamiento para regular los flujos de catalizador, y válvulas tapón que regulan los flujos de las mezclas gaseosas. La expresión general para los flujos regulados por válvulas es:

$$
\begin{aligned}
& F=K f \sqrt{\Delta P} \\
& \text { y } \Delta P=P_{\text {in }}+\left(W_{s} g_{u} / A_{t}\right)-P_{\text {out }}
\end{aligned}
$$

donde $K$ y $f$ son la constante y la función característica de la válvula, $g_{u}$ es la constante gravitacional, $P_{\text {in }}$ y $P_{\text {out }}$ son las presiones de entrada y salida, y $A_{t}$ es la área de sección transversal.

\section{MÉTODO DE SOLUCIÓN NUMÉRICA}

La solución del modelo se ha realizado usando una estructura modular acorde con las distintas etapas del proceso. Como resultado de las suposiciones tomadas en el modelo, las ecuaciones de conservación se reducen a sistemas de ecuaciones diferenciales-algebraicas (EDA). La subrutina DASSL (Brenan et al., 1989) es utilizada para resolver tales sistemas. Las fases gaseosas del tubo elevador y el regenerador se modelan como flujo pistón en estado pseudoestacionario lo cual genera sistemas EDA como función de la posición axial, i.e. un $\operatorname{EDA}(z)$. Por su parte, la fase sólida en el reactor-agotador y el regenerador se modela como tanque perfectamente agitado con variables dependientes del tiempo lo que genera un sistema diferencial ordinario (EDO) con respecto al tiempo, i.e. EDO $(t)$. 
Al iniciar una simulación se proporcionan las dimensiones de la UFCC, las características de las corrientes y condiciones de operación del caso en estudio (Tablas 2 y 3). Las fases gaseosas en el tubo elevador y regenerador se consideran en estado pseudoestacionario, por lo cual se resuelven los sistemas $\operatorname{EDA}(z)$ del tubo elevador y el regenerador para generar las condiciones iniciales para el sistema $E D O(t)$ del reactor y el regenerador. Una vez resuelto el sistema $E D O(t)$ para el primer incremento en el tiempo $\left(\Delta t_{i}\right)$, se actualizan las variables del tubo elevador, agotador y regenerador, y se evalúa el siguiente incremento en el tiempo $\Delta t_{i+1}$ hasta alcanzar el tiempo de simulación deseado.

Tabla 2: Dimensiones de la UFCC.

\begin{tabular}{l|r}
\hline \multicolumn{2}{l}{ Tubo elevador } \\
\hline Longitud, $\mathrm{m}$ & 40.0 \\
Diámetro transversal, $\mathrm{m}$ & 1.3 \\
\hline \multicolumn{2}{l}{ Reactor-agotador } \\
\hline Diámetro transversal, $\mathrm{m}$ & 6.0 \\
Altura, m & 3.1 \\
Inventario de catalizador, Ton & 65 \\
\hline \multicolumn{2}{|l}{ Regenerador } \\
\hline Diámetro transversal, m & 10.2 \\
Altura de los ciclones, $\mathrm{m}$ & 12.0 \\
Inventario de catalizador, Ton & 185 \\
\hline
\end{tabular}

Tabla 3. Características de las corrientes FCC.

\begin{tabular}{|c|c|c|}
\hline \multicolumn{3}{|l|}{ Alimentación } \\
\hline \multirow{3}{*}{\multicolumn{2}{|c|}{$\begin{array}{l}\text { Densidad específica @ 20/4 } \mathrm{C} \\
\text { Índice de refracción @ 20 C } \\
\text { Azufre, \% peso }\end{array}$}} & 0.9052 \\
\hline & & 1.5072 \\
\hline & & 2.0 \\
\hline \multirow[t]{3}{*}{ ASTM D1160, K } & $10 \% \mathrm{vol}$ & 595 \\
\hline & $50 \%$ & 693 \\
\hline & $90 \%$ & 792 \\
\hline \multicolumn{3}{|l|}{ Catalizador } \\
\hline \multirow{2}{*}{\multicolumn{2}{|c|}{$\begin{array}{l}\text { Actividad MAT en equilibrio, } \% \\
\text { Volumen de poro, } \mathrm{g} / \mathrm{cm}^{3}\end{array}$}} & 67 \\
\hline & & 0.28 \\
\hline \multicolumn{2}{|c|}{ Tamaño del cristal de zeolita, $\mu \mathrm{m}$} & 1.3 \\
\hline
\end{tabular}

\section{RESULTADOS Y DISCUSIÓN}

Con el fin de estudiar el efecto de condiciones de operación extremas del proceso, se seleccionaron dos casos nominales de estudio, ver Tabla 4. El primer caso se refiere a la operación en modo de combustión parcial del coque. La cantidad de aire suministrada es entonces menor a la relación estequiométrica de $\mathrm{O}_{2}$ necesaria para llevar a cabo las reacciones de combustión y por tanto el $\mathrm{O}_{2}$ se agota completamente antes de abandonar el regenerador. Por su parte, el segundo caso consti- tuye la operación más común donde se suministra aire en exceso para asegurar la combustión del coque hacia $\mathrm{CO}_{2}$. Debe mencionarse que ambos casos de estudio ocurren en la misma unidad y, para el presente estudio, la selección de los casos es independiente de los objetivos de producción. En particular, el primer caso de estudio se ha escogido porque la multiplicidad mayor a 3 estados estacionarios ha sido observada únicamente en combustión parcial de coque (Arbel et al., 1995). Una discusión amplia sobre el efecto de las condiciones de operación en las variables puede encontrarse en un estudio previo (Hernández-Barajas et al. 2006).

Tabla 4: Operación nominal de casos de estudio.

\begin{tabular}{|c|c|c|}
\hline Tipo de combustión & Parcial & Total \\
\hline Tubo elevador & & \\
\hline $\mathrm{F}$ de alimentación, $\mathrm{kg} / \mathrm{s}$ & 51 & 51 \\
\hline Presión, bar & 2.62 & 2.80 \\
\hline T de alimentación, $\mathrm{K}$ & 483 & 483 \\
\hline CATOIL, kg/kg & 9.1 & 8.4 \\
\hline Productos, \% peso Gasolina & 46.1 & 50.3 \\
\hline Gases & 12.5 & 17.4 \\
\hline Coque & 7.1 & 5.8 \\
\hline $\mathrm{T}$ de productos, $\mathrm{K}$ & 768 & 780 \\
\hline Reactor-agotador & & \\
\hline Presión, bar & 2.46 & 2.64 \\
\hline $\mathrm{T}$ de productos, $\mathrm{K}$ & 808 & 781 \\
\hline $\mathrm{F}$ de vapor, $\mathrm{kg} / \mathrm{s}$ & 1.2 & 1.2 \\
\hline $\mathrm{T}$ del vapor, $\mathrm{K}$ & 573 & 573 \\
\hline Regenerador & & \\
\hline $\mathrm{F}$ de aire, $\mathrm{kg} / \mathrm{s}$ & 31.9 & 39.9 \\
\hline $\mathrm{T}$ del aire, $\mathrm{K}$ & 430 & 430 \\
\hline $\mathrm{T}$ de lechos denso/diluido, $\mathrm{K}$ & 925/925 & 961/981 \\
\hline$\chi_{\text {reg }}, \mathrm{kg} / \mathrm{kg} \times 10^{2}$ & 0.21 & 0.02 \\
\hline gases, \% mol & 0.00 & 1.95 \\
\hline $\mathrm{CO}$ & 2.70 & 0.31 \\
\hline $\mathrm{CO}_{2}$ & 15.50 & 15.51 \\
\hline
\end{tabular}

\section{Casos nominales de operación}

La multiplicidad de estados estacionarios ha sido estudiada a partir de diagramas de calor generado-removido. El primer análisis realizado en este trabajo está relacionado al efecto del flujo de aire en la multiplicidad ya que la única condición de operación diferente en los dos casos de estudio es el flujo de aire (Fig. 2). Las intersecciones entre las curvas de calor constituyen los diversos estados estacionarios del proceso. La Fig. 2a muestra que existen 3 estados estacionarios para los dos casos. van Heerden (1953) estableció que un estado estacionario es estable si la derivada de la curva del calor generado es menor que la derivada de la curva de calor removido: 
$\frac{d Q_{\text {gen }}}{d T_{\text {den }}}<\frac{d Q_{\text {rem }}}{d T_{\text {den }}}$

en caso contrario, el estado estacionario es necesariamente inestable. Considerando la condición anterior, las intersecciones "A" y " $C$ " en la Fig. $2 a$ son estados estacionarios estables y la intersección "B" es inestable. Debido a que las UFCC operan a temperaturas altas para fomentar la conversión, entonces el estado estable "C" carece de valor operacional.

La estimación de 3 estados estacionarios coincide con los resultados de Arandes y de Lasa (1992) y Arbel et al. (1995). La Fig. 2a y Tabla 4 indican que el caso de combustión parcial presenta un estado estacionario estable superior a temperaturas de lecho denso menores que en combustión total. Nótese que la curva de calor generado presenta diversas inflexiones causadas por la ausencia de $\mathrm{O}_{2}$ en el intervalo de temperatura de $820-1020 \mathrm{~K}$, ver Fig. 2b. El caso de combustión completa no presenta tales inflexiones ya que el $\mathrm{O}_{2}$ nunca se agota en todo el intervalo de temperatura y por tanto, la curva de calor generado no muestra inflexiones abruptas.

Una vez que se ha estudiado la multiplicidad para los casos nominales, en las secciones siguientes se muestran las condiciones extremas de operación. Para tal fin, se emplean los dos casos de estudio y se variarán las condiciones de operación para simular tres escenarios: operación sin fuente externa de calor, operación con fluidización deficiente y operación con baja cantidad de promotores de combustión.

\section{Operación sin fuente externa de calor}

En las refinerías, el precalentamiento de la alimentación y el aire es una práctica común ya que proporcionan grados de libertad adicionales que permiten mayor flexibilidad en la operación y control. Sin embargo, la ausencia de precalentamiento puede causar que las reacciones del proceso no se lleven a cabo.

La Fig. 3 muestra la consecuencia de no considerar precalentamiento de tales corrientes, para el caso de combustión parcial. Nótese que, cuando la velocidad de combustión del coque es igual al valor originalmente reportado, no existe algún estado estacionario estable en la región típica de operación. Aunque la región típica de operación depende del ob- jetivo de producción, puede considerarse que las temperaturas del lecho denso oscilan entre 900 y $1020 \mathrm{~K}$. Si fuera posible aumentar la velocidad de combustión, ésta tendría que incrementarse al 230\% para obtener estados estacionarios, aunque éstos no estarían dentro de la región típica.
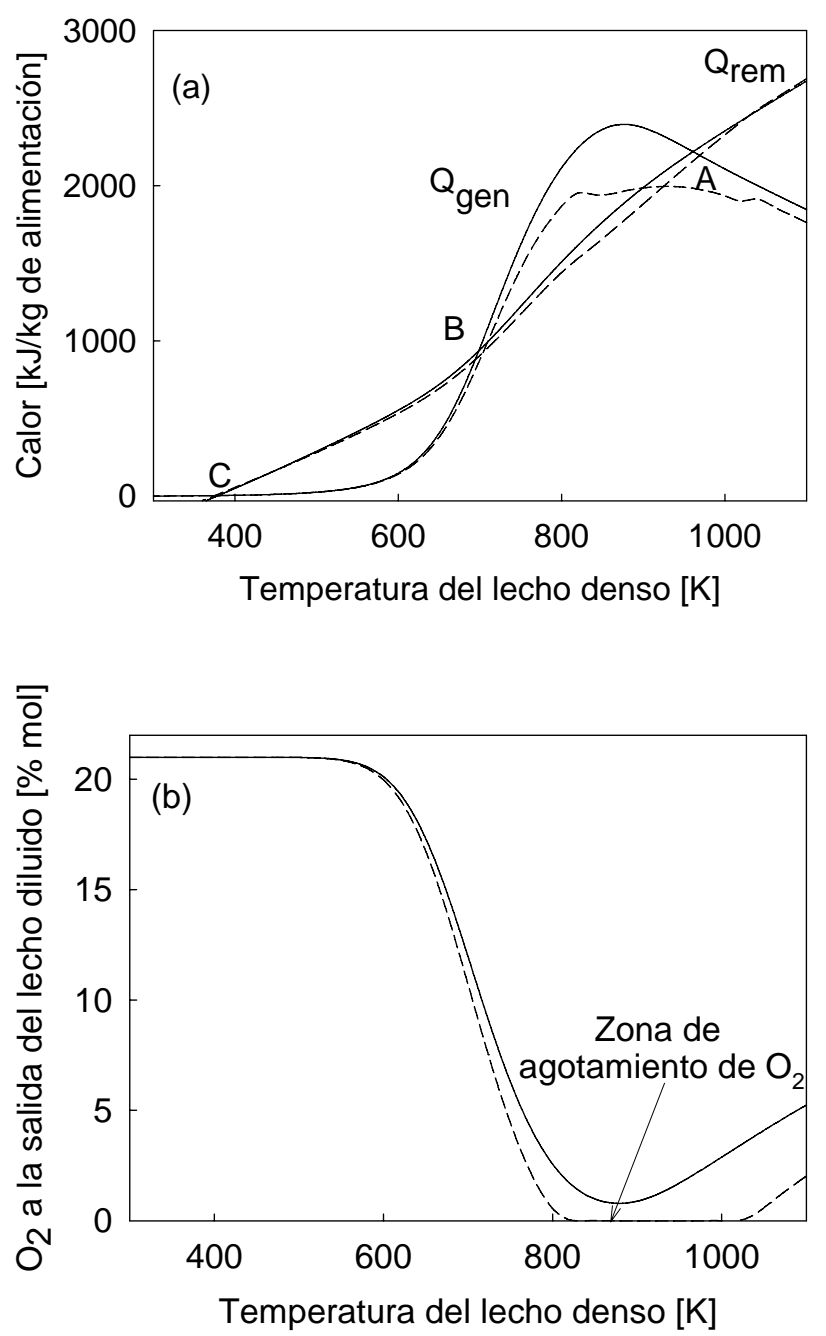

Fig. 2: Efecto del flujo de aire en la multiplicidad. En la figura, ----- Parcial, — Total.

\section{Operación con fluidización deficiente}

Uno de los problemas más comunes en la operación de unidades FCC está asociado con una deficiente fluidización del catalizador en el lecho denso del regenerador. En este estudio, tal deficiencia debe interpretarse como una distribución ineficiente del aire en el lecho. En consecuencia, las resistencias a la transferencia de masa y energía son mayores. En tales casos, la velocidad efectiva de combustión del coque es mucho menor al valor reportado en literatura. Debe recordarse que los parámetros cinéticos reportados en

Información Tecnológica - Vol. 18 No 1 - 2007 
literatura han sido obtenidos bajo condiciones de laboratorio donde la distribución de aire es prácticamente perfecta. En ese contexto, la Fig. 4 presenta el diagrama de calor del caso de combustión parcial para dos valores de la velocidad de combustión. Puede notarse que, cuando la velocidad de combustión es igual al $10 \%$, el proceso exhibe únicamente un estado estacionario. Contrariamente, si la velocidad de combustión es igual al $15 \%$, el proceso muestra los 3 estados estacionarios típicos.

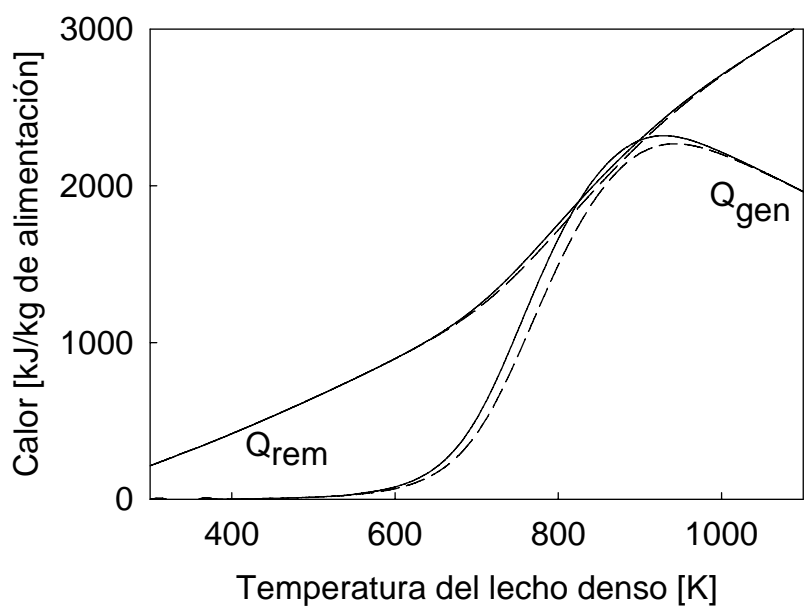

Fig. 3: Multiplicidad existente sin precalentamiento de las corrientes del proceso. En la figu-

$$
\text { ra, }-----R_{\chi}=100 \% \text {, }-R_{\chi}=230 \% \text {. }
$$

Simulaciones adicionales muestran que el cambio en la multiplicidad ocurre cuando $R_{\chi}=11 \%$. Una incertidumbre que surge es si el proceso puede mantenerse operando en el estado estacionario estable a estas condiciones. Para conocer la respuesta, se ha construido un diagrama plano-fase en donde pueden observarse las trayectorias dinámicas y los dominios de atracción de cada estado estacionario. La Fig. 5 muestra tal diagrama en donde la velocidad de combustión es igual a $11 \%$. Es notorio que el dominio de atracción del estado estable superior (y de interés industrial) es muy pequeño y se encuentra sumamente cercano al estado inestable. Lo anterior implica que la operación industrial en el estado estacionario requiere de una estrategia de control robusto que impida que el proceso migre hacia el estado intermedio y, en el peor escenario, los operadores puedan perder el control del proceso (ver Aguilar et al., 2002).

Además, se ha analizado el efecto de las velocidades bajas de combustión para el caso de combustión total, ver Fig. 6. Los resultados numéricos indican que cuando la velocidad de combustión es disminuida, el sistema presenta un único estado estacionario, tal como había ocurrido en el caso de combustión parcial. La diferencia principal es que ahora ocurre cuando la velocidad de combustión es igual al 22\%. Resultados posteriores han mostrado que el cambio en el número de estados estacionarios se presenta cuando $R_{\chi}$ $=30 \%$.

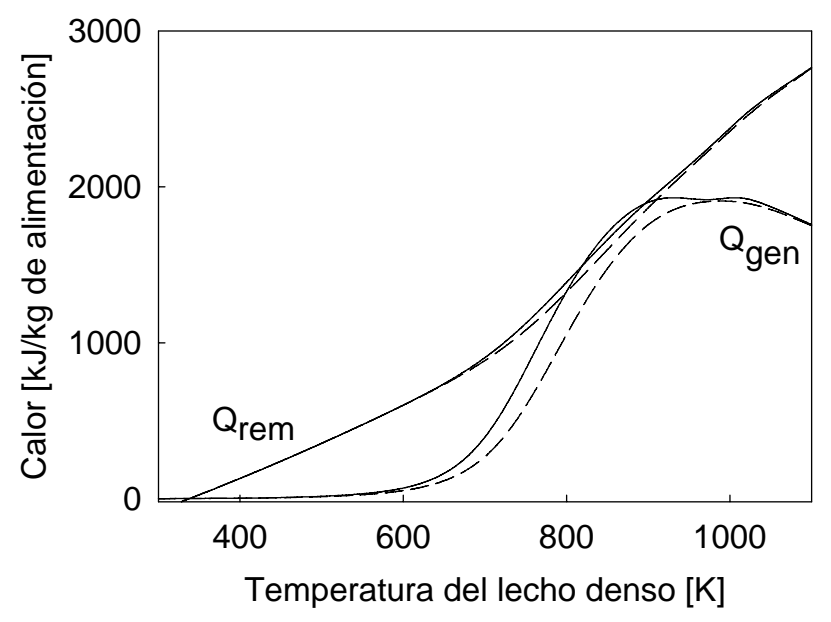

Fig. 4: Efecto de velocidades bajas de combustión de coque sobre la multiplicidad (combustión parcial). En la figura, ----- $R_{\chi}=10 \%$,

$$
R_{\chi}=15 \% \text {. }
$$

\section{Operación con baja cantidad de promotores}

En la formulación de catalizadores FCC suelen adicionarse promotores que fomentan la conversión de $\mathrm{CO}$ a $\mathrm{CO}_{2}$. Sin embargo, algunas refinerías disminuyen la adición de catalizador fresco para disminuir la conversión total de CO y utilizar el calor producido por esta oxidación en sistemas de recuperación de energía. En ese caso, la velocidad efectiva de oxidación heterogénea disminuye drásticamente. Este efecto es estudiado en este trabajo. La Fig. 7 muestra el análisis respectivo para ambos casos de estudio. Puede observarse que una disminución en la velocidad de oxidación implica la posibilidad de 5 estados estacionarios. Los estados estacionarios " $A$ ", "C" y "E" son estables mientras que los estados "B" $y$ "D" son inestables.

Los estados estacionarios de interés industrial son los 3 estados estacionarios superiores. El estado estacionario "A" ocurre a $1060 \mathrm{~K}$, una temperatura muy elevada y que se encuentra fuera del intervalo deseable de operación ya 


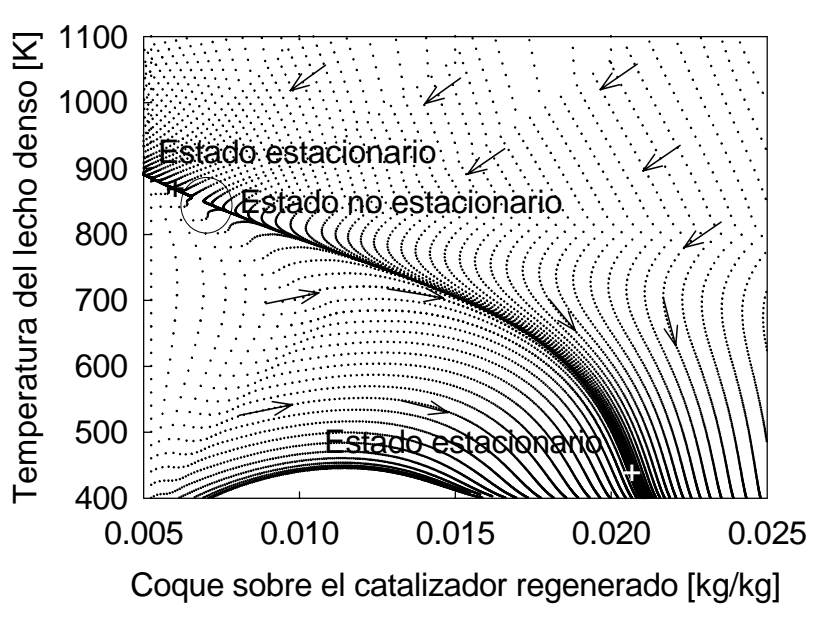

Fig. 5: Diagrama plano-fase mostrando 3 estados para $R_{\chi}=11 \%$ del valor original. En la figura, $\mathrm{EE}=$ estado estacionario y $\mathrm{ENE}=$ estado no estacionario.

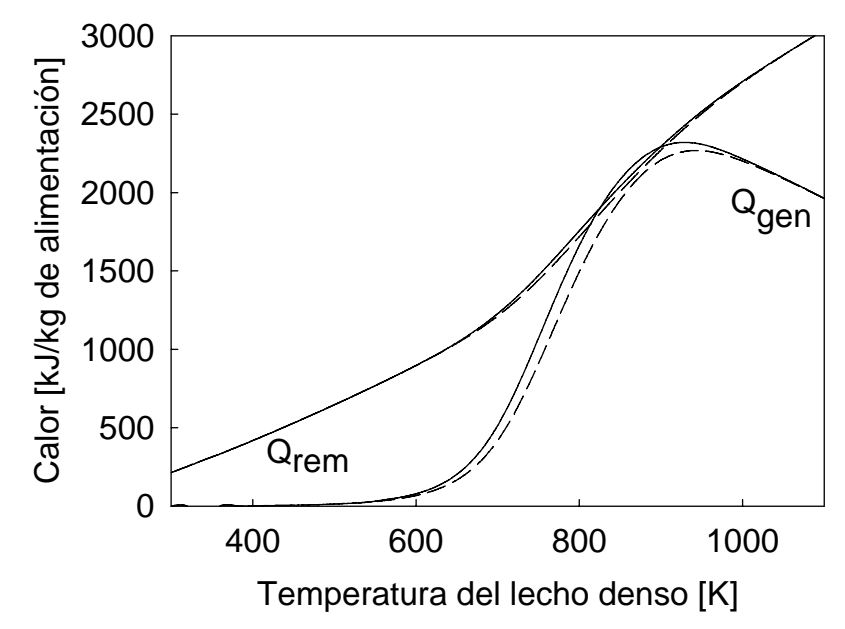

Fig. 6: Efecto de velocidades bajas de combustión de coque sobre la multiplicidad (combustión total). En la figura, ----- $R_{\chi}=22 \%,-R_{\chi}=32 \%$.

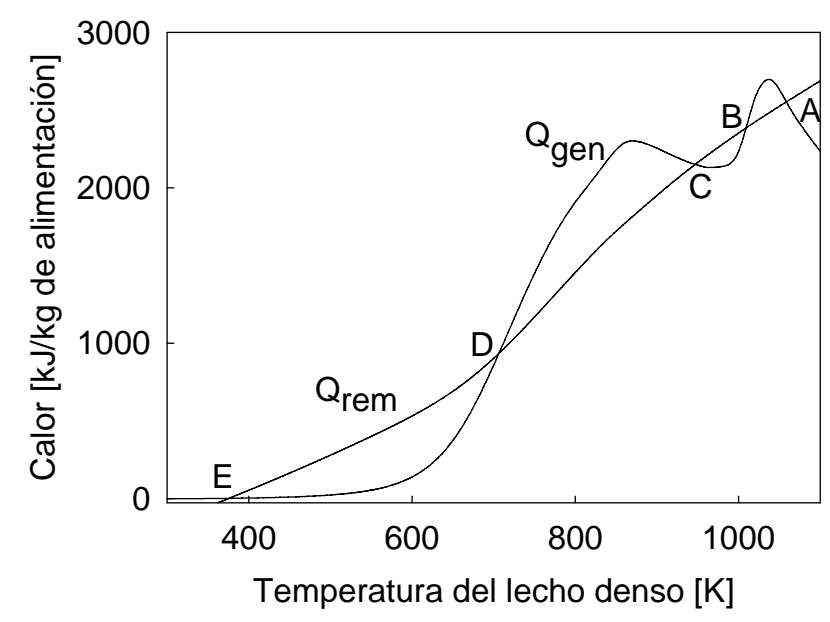

Fig. 7: Multiplicidad causada por una disminución de la velocidad catalítica de oxidación del $\mathrm{CO}_{2}$. que a tales temperaturas algunos equipos accesorios del proceso pueden sufrir daños metalúrgicos irreversibles. Por su parte, el estado estacionario "B" existe a $1010 \mathrm{~K}$, aún dentro del intervalo de operación pero se trata de un estado inestable. El estado estacionario "C" ocurre a una temperatura aceptable de $950 \mathrm{~K}$ y sobre la cual sería deseable la operación de la unidad cuando ocurra una disminución en la velocidad de oxidación del CO.

\section{CONCLUSIONES}

El efecto de condiciones extremas de operación en la multiplicidad de estados estacionarios para el proceso FCC fue analizado en este estudio. Los resultados muestran 3 estados estacionarios típicos de los cuales uno es inestable situado entre los otros dos estados que son estables. Sin embargo, a condiciones extremas pueden encontrarse de 1 a 5 estados estacionarios. La presencia de un solo estado estacionario se debe a la disminución abrupta en la eficiencia de la fluidización y la distribución del aire en el lecho denso del regenerador. Para ese caso, se determinaron los dominios de atracción de cada estado estacionario sugiriéndose que la proximidad entre los estados estacionarios sea tomada en cuenta en el diseño de una estrategia de control. La existencia de 5 estados estacionarios se relaciona con una disminución de la velocidad de oxidación del CO causada por una reducción en la concentración de promotores de combustión.

\section{NOMENCLATURA}

A Factor de frecuencia, $\mathrm{s}^{-1}$

C Concentración $\left[\mathrm{kgmol} / \mathrm{m}^{3}\right]$

E Energía de activación, [kJ/kgmol]

$F_{a} \quad$ Flujo de aire [kg/s]

$F_{g} \quad$ Flujo de gas $[\mathrm{kg} / \mathrm{s}]$

$F_{S} \quad$ Flujo de catalizador [kg/s]

$F_{v} \quad$ Flujo de vapor de agotamiento [kg/s]

$H \quad$ Entalpía [kJ/kg]

$k_{i j} \quad$ Constante cinética

M Peso molecular [kg/kgmol]

$n \quad$ Orden de reacción

$N \quad$ Número de moles [kgmol]

$\dot{Q} \quad$ Flujo volumétrico de gas $\left[\mathrm{m}^{3} / \mathrm{s}\right]$

$R_{i} \quad$ Velocidad de reacción

$u_{g} \quad$ Velocidad superficial del gas [m/s]

$W_{g} \quad$ Inventario de gas $[\mathrm{kg}]$

$W_{s}$ Inventario de catalizador [kg]

$t \quad$ Tiempo [s] 


$\begin{array}{ll}T & \text { Temperatura [K] } \\ y_{i} & \text { Fracción masa } \\ z & \text { Eje longitudinal }[\mathrm{m}]\end{array}$

Letras griegas

\author{
Densidad $\left[\mathrm{kg} / \mathrm{m}^{3}\right]$ \\ Fracción volumétrica $\left[\mathrm{m}^{3} / \mathrm{m}^{3}\right]$ \\ Función de desactivación catalítica \\ Contenido de coque $[\mathrm{kg} / \mathrm{kg}]$
}

\section{AGRADECIMIENTOS}

Los autores agradecen el apoyo brindado por la DGEST, CONCYTEG, CONACYT y PIFI.

\section{REFERENCIAS}

Ali, H. y S. Rohani, Dynamic Modeling and Simulation of a Riser-Type FCC Unit, Chem. Eng. Tech., 29, 118-130 (1997).

Aguilar, R., Poznyak, A., Martínez, R. y R. Maya. Tem-perature control in catalytic cracking reactors via a robust PID controller, J. Process Control, 12, 695-705 (2002).

Álvarez-Ramírez, J., Valencia, J. y H. Puebla. Multivariable control configurations for composition regulation in a fluid catalytic cracking unit, Chem. Eng. J., 99, 187-201 (2004).

Arandes, J. y H. de Lasa, Simulation and Multiplicity of Steady States in Fluidized FCCUs, Chem. Eng. Sci., 47, 2535-2540 (1992).

Arbel, A., Rinard I.H. y R. Shinnar, Dynamics and Control of Fluidized Catalytic Crackers. 2. Multiple Steady States and Instabilities, Ind. Eng. Chem. Res., 34, 3014-3026 (1995).

Brenan, K.E., S.L. Campbell y L.R. Petzold, "Numerical Solution of Initial-Value Problems in Differential-Algebraic Equations", $1^{\circ}$ edición, Elsevier Sci. Publishing, USA (1989).

Buchanan, J.S., Analysis of Heating and Vaporization of Feed Droplets in FCC Risers, Ind. Eng. Chem. Res., 33, 3104-3111 (1994).

Elnashaie, S.S. y I.M. El-Hennawi, Multiplicity of the Steady State in Fluidized Bed ReactorsIV. Fluid Catalytic Cracking (FCC), Chem. Eng. Sci., 34, 1113-1121 (1979).
Han I. y C. Chung, Dynamic Modeling and Simulation of a FCC Process. Part II, Chem. Eng. Sci., 56, 1973-1990 (2001).

Hernández, R., Vázquez, R. y D. Salazar. Un modelo acoplado para unidades de desintegración catalítica en lecho fluidizado, Información Tecnológica, 12(5), 123-129 (2001).

Hernández-Barajas, J.R., Vázquez-Román, R. y D. Salazar-Sotelo. Multiplicity of steady states in FCC units: effect of operating conditions, Fuel, 85, 849-859 (2006).

Hovd, M. y S. Skogestad. Procedure for Regulatory Control Structure Selection with Application to the FCC Process, AIChE J., 39(12), 1938-1953 (1993).

Howard, J.B., G.C. Williams y D.H. Fine, Kinetics of CO Oxidation in Postflame Gases, Proc. $14^{\text {th }}$ Symp. Int. Comb., 975-986 (1973).

Iscol, L., "The Dynamics of a Fluid Catalytic Crackers", Presentado en ASME $11^{\text {th }}$ Joint Aut. Control Conference, Atlanta, USA (1970).

Krishna, A.S. y E.S. Parkin, Modeling the Reg. in Commercial Fluid Catalytic Cracking Units, Chem. Eng. Prog., 81, 57-62 (1985).

Lee, W. y A.M. Kugelman, Number of SteadyState Operating Points and Local Stability of Open-Loop Fluid Catalytic Cracker, Ind. Eng. Chem. Process Des. Develop., 12(2), 197-204 (1973).

Morley, K. y H.I. de Lasa, On the Determination of Kinetic Parameters for the Regeneration of Cracking Catalyst, Can. J. Chem. Eng., 65, 773-777 (1987).

Schindler H. y H.I. de Lasa, "Regeneration of Coked Cracking Catalyst in a Pulse Microcatalytic Reactor", Proc. Can. Soc. Chem. Eng., 566-572, Quebec, Canadá (1984).

van Heerden, C., Autothermic Processes. Properties and Reactor Design, Ind. Eng. Chem., 45(6), 1242-1247 (1953).

Yen, L., "Kinetic Modelling of Fluid Catalytic Cracking", The AIChE Spring Nat. Meet., Abril 2-6, Houston, USA (1987). 